\title{
The acromion thoracic flap: A reconstructive resource after exeresis of a parotid fibrosarcoma
}

\author{
Mireya Bonet ${ }^{1}$, Enrique Zapater ${ }^{1}$, José R. Alba ${ }^{1}$, Jorge Basterra ${ }^{1}$ \\ ${ }^{1} \mathrm{MD}, \mathrm{PhD}, \mathrm{MD}, \mathrm{PhD}$. ENT Department. Valencia University General Hospital. Valencia Medical School. Spain
}

Correspondence:

C/ Baladre $n^{\circ} 3$ piso $1^{\circ}$ puerta 2 ,

46018 Valencia (Spain)

mireyabonet@gmail.com

\author{
Bonet M, Zapater E, Alba JR, Basterra J. The acromion thoracic flap: A \\ reconstructive resource after exeresis of a parotid fibrosarcoma. Med Oral \\ Patol Oral Cir Bucal. 2011 May 1;16 (3):e397-9. \\ http://www.medicinaoral.com/medoralfree01/v16i3/medoralv16i3p397.pdf

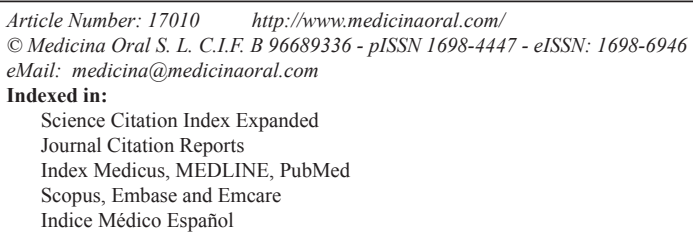

Received: $25 / 03 / 2010$

Accepted: 29/05/2010

\begin{abstract}
Sarcomas are rather uncommon in the salivary glands and have a high tendency towards recurrence. The treatment of choice is surgery, with the first surgical procedure being crucial, as the small size of the tumor is accompanied by the absence of post-surgery and post-radiotherapy fibrosis, which facilitates the surgery. The evident tendency towards recurrence makes it advisable to be radical in the exeresis. Often we have to consider a reconstructive period. Negative surgical margins increases the rates of local control. Treatment with radiotherapy is the complementary treatment indicated for patients with surgical margins less than $2 \mathrm{~mm}$ or positive. It has been observed that this treatment improves the local control of the illness. We present a case of parotid fibrosarcoma of a low grade of malignancy that was treated by means of parotidectomy and post-operative radiotherapy. The patient presented up to three recurrences, one of which required an acromion thoracic flap in order to reconstruct the surgical defect. Despite the treatment, the patient ended up dying because one of the recurrences was not operable.
\end{abstract}

Key words: Acromion thoracic flap, fibrosarcoma, parotid tumours, radiotherapy.

\section{Introduction}

Malignant tumors located in the salivary glands are most often epithelial tumors. Non-epithelial tumors account for less than $5 \%$ of the tumors in this location, $90 \%$ of which are benign. Sarcomas are malignant non-epithelial tumors; in the head and neck, they are a nosological entity that occurs relatively infrequently, with those of the soft parts of the neck and those of the parotid gland are the most common (1-3). Microscopically, this types of tumors showed an intense proliferation of spindle- shaped cells, varying a little in size and shape and arranged in parallel bands, partly crossing each other (4). The diffe- rent histological types can be classified according to their high or low grade of malignancy (5). Fibrosarcoma of the head and neck represents $5 \%$ of all fibrosarcomas.

Present the case of a patient with a low-grade recurrent parotid fibrosarcoma which required various surgical procedures, one of which required a regional flap for the reconstruction following exeresis.

\section{Case Report}

A 69-year-old patient comes in for a visit to the doctor due to the presence of a tumor located in the right parotid gland, which has been evolving over the past 
5 months and has experienced rapid growth in the last month. An elastic and mobile lesion is observed, which can be delimited upon touch, measuring approximately $3 \mathrm{~cm}$. No facial paralysis is detected. A Fine Needle Aspiration (FNA) examination shows the existence of fibroadipose cells without visible signs of malignance. The MRI shows a well-defined hyperintense lesion in $\mathrm{T} 1$, located in the superficial lobe of the parotid gland, and which does not enhance after gadolinium. The radiology report suggests the diagnosis of lipoma. We decide surgical treatment, consisting in superficial parotidectomy with an extemporaneous anatomopathological study. The pathologist informs of a malignant lesion, without being able to specify the histological origin. The parotidectomy is completed by resecting the deep lobe. No signs of invasion of the facial nerve are observed; thus, given the clinical lack of involvement of the facial nerve, it is left intact. The definitive anatomopathological study results in a diagnosis of low-grade malignant fibrosarcoma, detecting infiltration of one of the edges of resection of the surgical piece, and thus, we decided to administer post-operative radiotherapy.

Fourteen months after the surgery, a lesion measuring 4 $\mathrm{cm}$ is observed in the area of the mandibular angle, with a firm consistency although non-petrous, which appears to be attached to deep surfaces and to the skin (Fig. 1). The tumor had grown during the last month, the CT image (Fig. 2a), after applying contrast, shows an irregular hypodense lesion with an infiltrative appearance which enters into contact with the anterior side of the sternocleidomastoid muscle. A slight ballooning of the floor of the mouth and the oropharynx and hypopharynx are detected. In the MRI (Fig. 2b), a significant amount of gadolinium surrounding the ascending branch of the mandible and extending up to the area of the temporomandibular joint is identified.

We decide surgical treatment performing an exeresis as requested, including facial skin, anterior cartilaginous wall of the outer ear canal and the earshell. The internal jugular vein, external carotid artery, sternocleidomastoid muscle and cranial pairs VII, IX, X, XI and XII are resected. The lesion is in very close contact with the ascending branch of the mandible, which is resected from the mandibular angle up to the condyle, both included. In order to reconstruct the surgical defect, an acromion thoracic flap is made. This flap has a rectangular shape, with an upper incision that begins in the medial third of the clavicle and runs parallel to it towards the sternum and then descends vertically until approximately the fifth intercostal space, where it runs horizontal and goes up to the anterior axilar line and deltoid muscle (Fig. 3a, 3b, 3c) to cover the donor area, a graft of loose skin is used (6).

The pathological study confirms the existence of a lowgrade fusocellular sarcoma that involves the skin, adi-

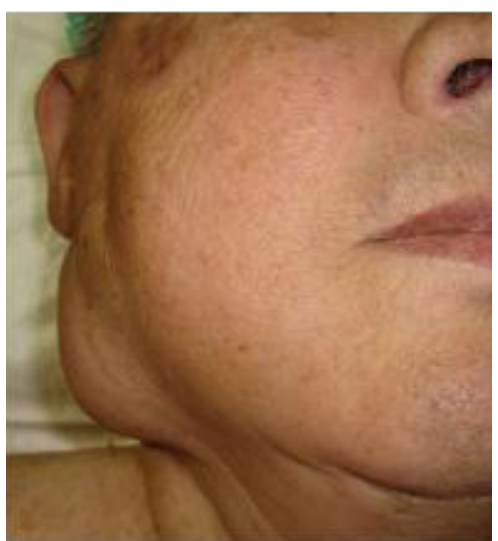

Fig. 1. Frontal view of the tumor located in the topography of the parotid gland that the patient presented the first time that the disease was shown to be recurrent.

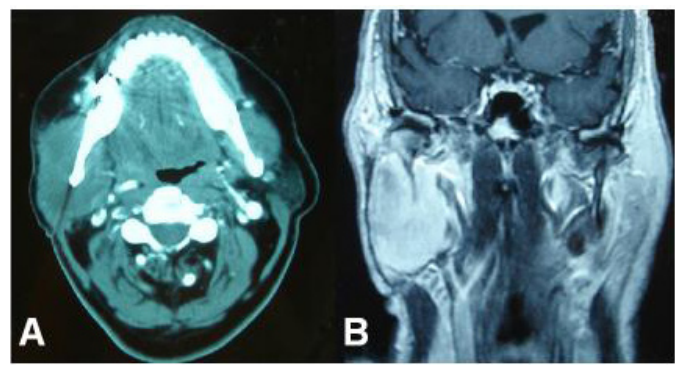

Fig. 2a. CT image after applying a contrast, which shows in the topography of the right masseter, right parotid gland and internal pterygoid an irregular hypodense lesion with an infiltrating appearance that causes swelling of the fat and skin in the parotid region. This lesion comes into contact with the anterior side of the sternocleidomastoid muscle, respecting the cervical vessels. A slight swelling of the oropharynx is observed, which causes asymmetry of the aerial light. Fig. 2b. Coronal image from the MRI after injection of gadolinium. A large contrasting mass is identified, which surrounds the branch that travels up the mandible until the area of the temporomandibular joint; moves to the internal and external carotid arteries and to the internal jugular vein.

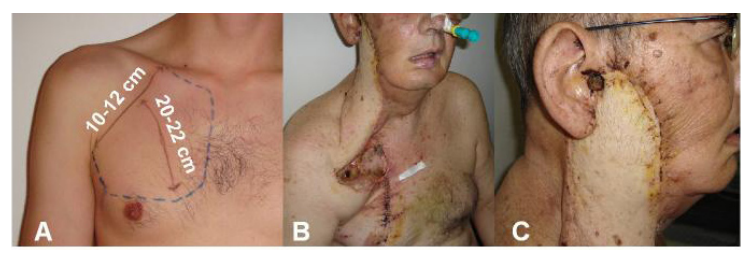

Fig. 3a. Diagram of the design of the flap. Fig. 3b. Frontal view of the acromion thoracic flap used for reconstructing the defect arising after the surgical exeresis of the first recurrence (postoperative phase). Fig. 3c. Lateral view of said flap in which repair of the outer ear canal can be observed. 
pose tissue, sternocleidomastoid muscle and mandibular bone.

One year after the last surgical procedures, two lesions were detected. One was located in the same topography as the lesion that was operated one year prior, and the other is a right posterior cervical location. The image study by means of $\mathrm{CT}$ shows the presence of a lesion that occupies $7 \times 3 \mathrm{~cm}$ of space, located in the parapharynx, and which goes from the base of the skull to the hyoid bone. The lesion completely surrounds the internal carotid artery. Another $3 \times 3 \mathrm{~cm}$ lesion is observed, located in the subcutaneous fat next to the right splenius muscle.

We decide to perform another surgery, which initially involves a cervicotomy and exeresis of the posterior cervical lesion until exposing the scalenes and vertebral transverse apophysis. The anterior lesion completely surrounds the perimeter of the internal carotid artery, but can be resected, except in its upper end, where it comes into intimate contact with the base of the cranium.

Given that a complete resection is impossible, Oncology Committee decides to administer adjunct radiotherapy treatment on the affected area, at a dose of $60.4 \mathrm{~Gy}$.

Six months afterwards, another recurrence is detected. We decide palliative chemotherapy treatment. The patient dies nine months after initiating the chemotherapy treatment.

\section{Discussion}

The case presented shows the recurrent tendency of parotid fibrosarcoma, even when it involves a lesion with a low grade of malignance (7-9). Surgery is the primary treatment for these types of tumors (10). The first surgical procedure is crucial, as the small size of the tumor is accompanied by the absence of post-surgery and postradiotherapy fibrosis, which facilitates the surgery. In the presented case, knowing the evolution, we now consider what would have occurred if we had been more aggressive in the first surgical procedure, eliminating the facial nerve and increasing the margins of resection. We decided post-operative radiotherapy treatment, which, in the long run, proved to be ineffective.

The MRI did not give any indication that the lesion was malignant, which would have helped to plan the surgical procedure.

The appearance of the first recurrence was treated surgically with a required exeresis. During the procedure, our attention was drawn to the fact that the common and internal carotid arteries were easily detached from the tumor without it being necessary to access the subadventitial layer, despite being completely surrounded by the lesion. This tumor behavior is different from that which commonly occurs in epidermoid carcinomas. Usually, it is considered that when a metastatic adenopathy of an epidermoid carcinoma of the head and neck surrounds more than $270^{\circ}$ of the internal carotid artery, the artery presents an invasion of its wall (11).

In order to repair the defect produced following the resection, we performed an acromion thoracic flap. It is a randomized fasciocutaneous flap that is vascularized by the acromial branch of the thoracoacromial artery and the extensive vascular network of the thoracic region. It is a technically simple, although infrequent flap, which includes a large section of skin, subcutaneous cell tissue, mammary gland and fascia of the pectoralis major muscle.

Although radiotherapy is considered to be an important treatment option, the radiosensitivity of these tumors is questionable according to many authors (12). In our case, the evolution has not improved. Neo-adjunct radiotherapy is reserved for some recurrent and inoperable cases in patients in which the base of the cranium is involved (13-14).

Despite the multidisciplinary management of this type of tumor, failure of the treatment is not rare, and the case that we present is another proof of this bad prognosis.

\section{References References with links to Crossref-DOI}

1. Mendenhall WM, Mendenhall CM, Werning JW, Riggs CE, Mendenhall NP. Adult head and neck soft tissue sarcomas. Head Neck. 2005;27:916-22.

2. Barker JL Jr, Paulino AC, Feeney S, McCulloch T, Hoffman H. Locoregional treatment for adult soft tissue sarcomas of the head and neck: an institutional review. Cancer J. 2003;9:49-57.

3. Rapidis AD. Sarcomas of the head and neck in adult patients: current concepts and future perspectives. Expert Rev Anticancer Ther. 2008;8:1271-97.

4. Soares AB, Lins LH, Macedo AP, Pereira-Neto JS, Vargas PA. Fibrosarcoma originating in the mandible. Med Oral Patol Oral Cir Bucal. 2006;11:E243-6.

5. Sturgis EM, Potter BO. Sarcomas of the head and neck region. Curr Opin Oncol. 2003;15:239-52.

6. Conley JJ. The use of regional flaps in head and neck surgery. Ann Otol Rhinol Laryngol. 1960;69:1223-34.

7. Kraus DH. Sarcomas of the head and neck. Curr Oncol Rep. 2002;4:68-75.

8. Hammami B, Bouayed W, Siala W, Toumi N, Khabir A, Boudawara $\mathrm{T}$, et al. [Head and neck sarcoma]. Ann Otolaryngol Chir Cervicofac. 2008;125:294-300.

9. Chen SA, Morris CG, Amdur RJ, Werning JW, Villaret DB, Mendenhall WM. Adult head and neck soft tissue sarcomas. Am J Clin Oncol. 2005;28:259-63.

10. Penel N, Van Haverbeke C, Lartigau E, Vilain MO, Ton Van J, Mallet $\mathrm{Y}$, et al. Head and neck soft tissue sarcomas of adult: prognostic value of surgery in multimodal therapeutic approach. Oral Oncol. 2004;40:890-7.

11. Yousem DM, Hatabu H, Hurst RW, Seigerman HM, Montone KT, Weinstein GS, et al. Carotid artery invasion by head and neck masses: prediction with MR imaging. Radiology. 1995;195:715-20.

12. Laramore GE. Role of particle radiotherapy in the management of head and neck cancer. Curr Opin Oncol. 2009;21:224-31.

13. Pellitteri PK, Ferlito A, Bradley PJ, Shaha AR, Rinaldo A. Management of sarcomas of the head and neck in adults. Oral Oncol. 2003;39:2-12.

14. De Paoli A, Bertola G, Boz G, Gherlinzoni F, Frustaci S, Fumo $\mathrm{G}$, et al. Radiation therapy and conservative surgery for soft tissue sarcomas of the extremities, torso and head and neck. Ann Oncol. 1992;3 Suppl 2:S97-101. 